\title{
ARTICLE Angiotensin involvement in trauma processing - exploring candidate neurocognitive mechanisms of preventing post-traumatic stress symptoms
}

\author{
Lorika Shkreli ${ }^{1,2,3}$, Marcella Lydia Woud (iD ${ }^{2}$, Roger Ramsbottom ${ }^{4}$, Aleksandra Ewa Rupietta ${ }^{2,5}$, Gerd Thomas Waldhauser ${ }^{5}$, \\ Robert Kumsta (iD $^{6}$ and Andrea Reinecke ${ }^{3}$
}

The angiotensin-II antagonist losartan is a promising candidate that has enhanced extinction in a post-traumatic stress disorder (PTSD) animal model and was related to reducing PTSD symptom development in humans. Here, we investigate the neurocognitive mechanisms underlying these results, testing the effect of losartan on data-driven and contextual processing of traumatic material, mechanisms proposed to be relevant for PTSD development. In a double-blind between-subject design, 40 healthy participants were randomised to a single oral dose of losartan $(50 \mathrm{mg})$ or placebo, $1 \mathrm{~h}$ before being exposed to distressing films as a trauma analogue while heart rate (HR) was measured. Peritraumatic processing was investigated using blurry picture stimuli from the films, which transformed into clear images. Data-driven processing was measured by the level of blurriness at which contents were recognised. Contextual processing was measured as the amount of context information retrieved when describing the pictures' contents. Negative-matched control images were used to test perceptual processing of peripheral trauma-cues. Post-traumatic stress symptoms were assessed via self-report questionnaires after analogue trauma and an intrusion diary completed over 4 days following the experiment. Compared to placebo, losartan facilitated contextual processing and enhanced detail perception in the negative-match pictures. During the films, the losartan group recorded lower HR and higher HR variability, reflecting lower autonomic stress responses. We discuss potential mechanisms of losartan in preventing PTSD symptomatology, including the role of reduced arousal and increased contextual processing during trauma exposure, as well as increased threat-safety differentiation when encountering peripheral trauma-cues in the aftermaths of traumatic events.

Neuropsychopharmacology (2020) 45:507-514; https://doi.org/10.1038/s41386-019-0553-y

\section{INTRODUCTION}

Post-traumatic stress disorder (PTSD) is a highly prevalent and disabling condition with enormous economic impact $[1,2]$. Elucidating the interplay of cognitive and neurochemical mechanisms in disorder development would have tremendous implications for the identification of prevention and treatment targets, yet this relationship remains poorly understood. Recent research increasingly suggests involvement of the renin-angiotensin system in threat processing [3, 4] and anxiety [5-7]. Although angiotensin-II $\left(\mathrm{AT}_{1}\right)$ receptors are primarily associated with cardiovascular regulation, they are not only expressed in the periphery, but also in brain areas relevant to fear, including amygdala, hippocampus, and prefrontal cortex [8]. Receptor blockade leads to increased availability of angiotensin-IIconverted angiotensin IV, thought to be crucial for neuroplasticity [9]. Accordingly, studies have shown that the AT 1 receptor antagonist losartan improves fear extinction in mice [7] and is related to preventing the development of PTSD symptoms in humans if active during trauma [6]. However, the cognitive mechanisms of action underlying such effects remain to be identified, which would inform the development of prevention and treatment strategies for PTSD.

Cognitive models propose that PTSD develops due to an excess of perceptual, data-driven processing and a lack of contextual, deliberately retrievable information processing during trauma exposure [10-13]. As a consequence, stimuli perceptually similar to trauma characteristics automatically trigger trauma memories, resulting in re-experiencing symptoms such as intrusions [12, 13]. Accordingly, studies in healthy volunteers and trauma-survivors confirm a relationship between increased perceptual processing and re-experiencing symptoms [14-16]. Furthermore, experimental analogue studies suggest that predominantly using contextual versus data-driven processing during exposure to distressing material reduces re-experiencing symptoms $[17,18]$. This also corresponds with neurobiological models of PTSD suggesting an exaggerated amygdala response for low-level processing (i.e. datadriven processing) and damped responses in hippocampal-based high-level processing (i.e. contextual processing) $[10,13,19]$.

The present study aimed to elucidate potential neurocognitive mechanisms underlying the prevention of PTSD symptoms

\footnotetext{
${ }^{1}$ Department of Psychiatry, University of Cambridge, Cambridge, UK; ${ }^{2}$ Department of Clinical Psychology and Psychotherapy, Ruhr-Universität Bochum, Bochum, Germany

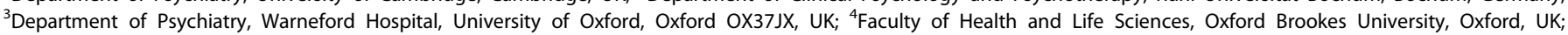
${ }^{5}$ Department of Neuropsychology, Ruhr-Universität Bochum, Bochum, Germany and ${ }^{6}$ Genetic Psychology, Ruhr-Universität Bochum, Bochum, Germany

Correspondence: Andrea Reinecke (andrea.reinecke@psych.ox.ac.uk)

These authors contributed equally: Lorika Shkreli, Marcella Lydia Woud
}

Received: 4 June 2019 Revised: 14 October 2019 Accepted: 17 October 2019

Published online: 26 October 2019 
through $\mathrm{AT}_{1}$ receptor blockade [6]. In a double-blind randomised design, we investigated the effect of a single dose of losartan versus placebo on data-driven and contextual processing of distressing films, an experimental analogue to a real-life traumatic event [20-22]. Since animal studies revealed decreased stress responses after injecting losartan into the amygdala [23] and enhanced hippocampus-driven memory and learning performance [24-26], we hypothesised that losartan would decrease data-driven processing and/or increase contextual processing. Further, we expected fewer post-traumatic stress symptoms in the losartan compared to the placebo group. Previous work also suggests that cardiovascular arousal plays an important role in PTSD symptom development [27]. In particular, clinical studies revealed that PTSD was associated with increases in heart rate (HR), as an indicator of psychophysiological arousal, shortly after trauma (i.e. in ambulance/emergency room after major burn injuries) [28] and in response to trauma-related stimuli [29]. Similarly, decreases in heart rate variability (HRV), as an indicator of elevated sympathetic activity relative to parasympathetic activity, were associated with PTSD diagnosis in veterans [30]. Interestingly, rodent work indicates that cardiovascular response to stress, including an accelerated $\mathrm{HR}$, might be regulated by $\mathrm{AT}_{1}$ receptor functioning [31,32]. We therefore conducted exploratory analyses to investigate the relationship between losartan and peritraumatic HR and HRV as an indicator of stress and arousal.

\section{MATERIAL AND METHODS}

\section{Participants}

Forty healthy participants (26 women) $(M=25.85, S D=6.89$ years) were recruited via study advertisements placed on public noticeboards (e.g., community centres, college noticeboards) or posted to local online classifieds, and were included if they were aged $18-50$, not pregnant, lactating or trying to get pregnant, had a body mass index of $18-30 \mathrm{~kg} / \mathrm{m}^{2}$, smoked $<5$ cigarettes/day, had not taken CNS-active medication in the last 6 weeks, had no firstdegree family history of a severe psychiatric disorder, did not meet criteria for any current psychiatric disorder, and never met diagnostic criteria for PTSD, bipolar, or psychotic disorders. Eligibility was determined through medical screening and standardised psychiatric screening (SCID-V; structured clinical interview for DSM-5) [33].

The following baseline measures were assessed: National Adult Reading Test [34], Eysenck Personality Questionnaire [35], Trauma History Checklist [20], Post-traumatic Cognition Inventory [36], Anxiety Sensitivity Inventory [37], Beck Depression Inventory II [38], Spielberger Trait Anxiety Inventory [39], Attention Control Scale [40], and Behavioural Inhibition and Activation Scale [41]. All participants gave written informed consent. The study was approved by University of Oxford ethics committee.

\section{Procedure}

The study involved two one-to-one visits and a follow-up assessment via mail. During a screening visit, eligibility was determined. Prior to the testing visit, participants were randomly allocated to either a single oral administration of $50 \mathrm{mg}$ losartan (Cozaar; Merck Sharp \& Dohme Ltd) or matched placebo (microcrystalline cellulose; Rayotabs, Rayonex $\mathrm{GmbH}$ ), stratified for sex. To ensure that losartan did not induce response bias, blood pressure and HR were measured before and $1 \mathrm{~h}$ after drug administration at expected peak level, and participants completed a state anxiety questionnaire (STAI-S; [39]) and visual analogue scales rating their mood and physiological symptoms (see Supplementary Material). Following this second assessment $(\sim 1 \mathrm{~h}$ after drug intake), double-blind testing started and took about $1 \mathrm{~h}$ 30 min $[42,43]$. This included watching the trauma films while HR response was monitored $(20 \mathrm{~min})$, and then performing the peritraumatic processing task (PPT) $(45 \mathrm{~min})$. Mood was measured pre-post trauma films (5 $\mathrm{min})$, and post-traumatic cognitions were measured using the Post-traumatic Cognition Inventory (PTCl; [36]) (5 min) following the film.

At the end of the testing session participants and experimenter also guessed whether the active capsule had been administered. Participants were then explained the concept of intrusions and instructed to record these over the next 4 days in an intrusion diary. Participants also received a sealed, pre-paid envelope with follow-up questionnaires (PTSD Checklist for DSM-5 (PCL-5) [44]; PTCl; explicit memory questionnaire, diary compliance form) which had to be filled in at day 4 .

\section{Trauma-film paradigm}

In line with previous analogue research (for review, see [45]), participants were presented a 19-min trauma film, comprising 26 distinctive clips. The clips varied between $20 \mathrm{~s}$ and $1 \mathrm{~min}$ and were based on the DSM-5-A criterion for PTSD [46], i.e., a person witnessing a traumatic event (Supplementary Material). Mood ratings were used pre- and post-films to verify that the trauma induction was successful $[22,47]$.

Peritraumatic HR and HRV during film

Potential drug effects on HR and HRV during analogue trauma exposure were explored using short-range telemetry (POLAR $\mathrm{RS} 800 \mathrm{CX}$ ). $\mathrm{R}$ to $\mathrm{R}$ intervals (RRl; milliseconds) were recorded for the duration of the intervention (losartan and placebo). The RRI data were checked for anomalies and non-sinus beats. Thereafter 256 continuous RRI was analysed for each participant under each condition. For statistical analyses, we calculated the mean HR and HRV (LF/HF ratio using frequency-domain results within Fast Fourier Transform spectrum; Kubios Software, HRV Standard (ver. 3.3.0)) for each participant (see [48]).

\section{Peritraumatic processing task}

Based on theories of PTSD development [11-13], this task was designed to simultaneously measure data-driven processing and contextual processing. Participants were presented blurry pictures one by one in a random order. Over a time-frame of $32 \mathrm{~s}$ the blurry pictures transformed into a clear image. Participants were instructed to press a key as soon as they recognised the contents of the picture. Participants then indicated whether the image was part of the trauma film or not, after which they typed in a brief description of the picture's contents. The task consisted of three practice trials (neutral images) and 52 test trials, including 26 pictures showing the hotspot of a previously seen trauma-film clip, and 26 negative-matched pictures of which 22 were IAPS [49] and 4 were non-IAPS images (Fig. 1) (see Supplementary Materials for more details on stimuli creation and Table S2 for overview of stimulus material and IAPS codes).

Data-driven processing. Data-driven processing was reflected in reaction times (RT), with faster response reflecting image recognition at blurrier picture levels during the PPT, similar to perceptual priming effects given the low-level sensory features at this stage $[15,16,50]$. This could be measured equally for trauma images and negative-match images, since these were matched based on low-level visual features.

Contextual processing. Contextual processing was assessed by the level of context detail given during the PPT in the description of the trauma-film pictures (Fig. 1). When describing the pictures, participants could include context information which was not depicted in the presented picture itself but which they deliberately retrieved based on their (potentially more detailed) memory of the shown scene. The level of detail given for negativematched pictures, however, reflected pure perceptual processes, since all pictures were new and no memory effects could interfere. Thus, it reflected general visual processing of peripheral 
A $\quad$ Up to $32 \sec$

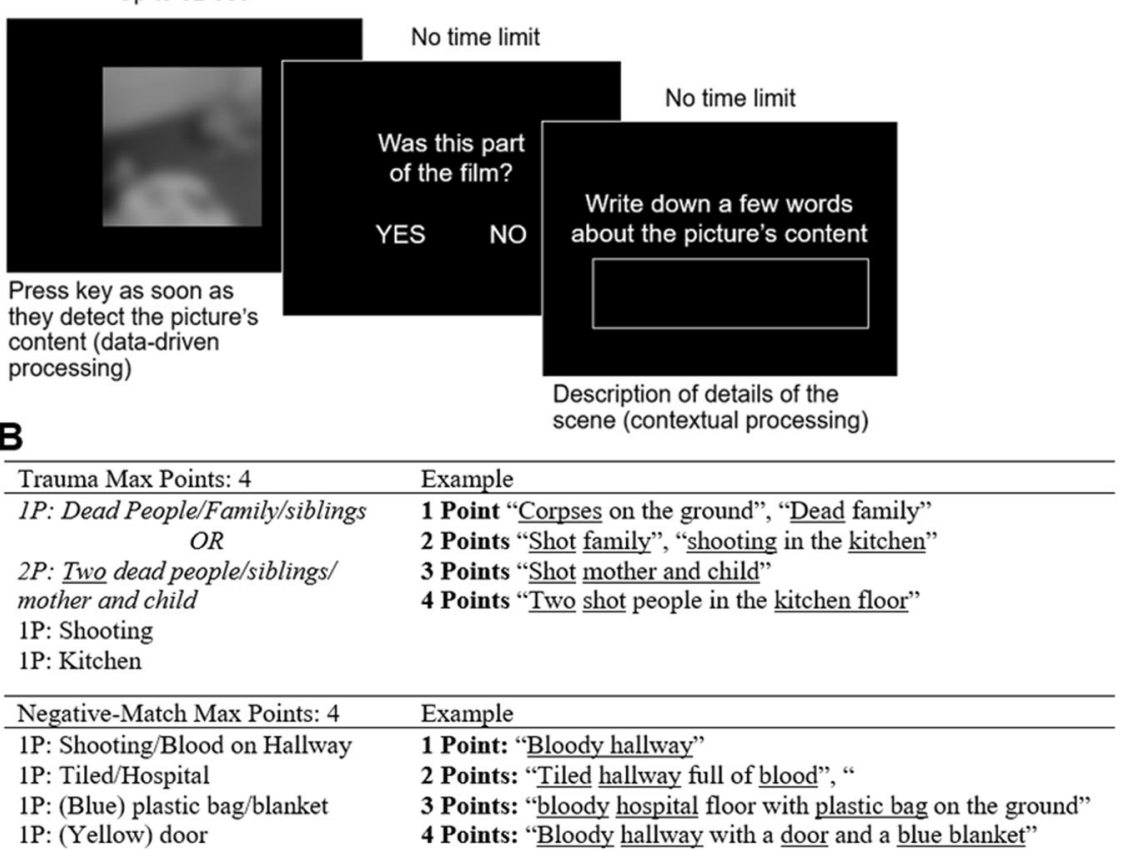

Fig. 1 Peritraumatic processing task and scoring system. a Paradigm overview. A trial consisted of three stages. First, a blurry picture increasingly transformed into the clear image, and participants pressed a key as soon as they thought they recognised the image content. Second, the picture was categorised as part of the trauma film or not. Third, participants described the picture's contents. Intertrial interval was jittered between 1.5 and $2.5 \mathrm{~s}$. b Scoring system. For each detail provided, participants received one point. For the trauma pictures, points were also given for details not presented in the picture but only seen in the film. Italic print indicates where only the higher value was considered for scoring, since it contained the information from the lower value. The full scoring scheme can be requested from the authors

trauma-cues, meaning stimuli that had not actually been experienced as part of the trauma film but matched elements of the trauma-film scene in key perceptual and conceptual features. To determine the level of details given, a scoring system was used where for both picture types, the number of details was predetermined, and participants were given one point per reported detail (for trauma pictures, reported details could also relate to information not presented in the picture). The number of points was added to a sum score per picture type, with the same possible total score across all pictures in both conditions. Two independent 'raters' scored the descriptions based on the a priori scoring system (interrater reliability: $r=0.913$; accordance $=89.7 \%$ ). The mean score of both raters was used for analyses.

\section{Assessment of post-traumatic stress symptoms}

Post-traumatic cognitions were measured using the PTCl after trauma-film presentation and at follow-up. Also, at follow-up the PCL-5 was administered as a measure of overall PTSD symptoms. Intrusive memories of the films were recorded using a pen-andpaper diary [20-22]. Intrusions were defined as mental images or verbal thoughts about the films' contents, which spontaneously occurred without deliberate retrieval. On the 4 days following the experiment, participants were instructed to record every intrusion they experienced and to give a description of the intrusion's content. Intrusion distress was recorded per intrusion $(0=$ not distressing at all to $100=$ extremely distressing). One day after diary completion we measured diary compliance via questionnaire [20-22] (Supplementary Material).

Explicit memory

To test explicit memory for trauma-film contents at follow-up, a multiple-choice question with one correct answer out of four was given for each trauma-film clip (for similar approaches [47, 51, 52]). The outcome measure was the number of correct answers.

\section{RESULTS}

Statistical analyses were conducted using SPSS software (IBM SPSS, Inc., Armonk NY) with an alpha-level of 0.05 on twotailed tests.

Sociodemographic and clinical characteristics

There were no acute drug effects of losartan on physiological and subjective mood markers that could induce response bias (for analyses and results, see Supplementary Material and Table S1). Independent-samples $t$-tests showed that groups were comparable on all sociodemographic, clinical, attentional, and personality measures (see Table 1).

Trauma film

Participants did not differ in state anxiety prior to film presentation (placebo: $\mathrm{M}=28.90$; $\mathrm{SD}=6.40$; losartan: $\mathrm{M}=30.05, \mathrm{SD}=7.47$; $t(38)=-0.52, p=0.604, d=0.17)$. Mood pre- and post-film was compared using mixed-model ANOVAs with the between-factor drug group (placebo, losartan) and the within-factor time (pre-film, post-film). Negative mood increased equally across both groups, suggesting successful analogue trauma induction $(F(1,38)=71.83$, $p<0.001, \eta_{\mathrm{p}}{ }^{2}=0.654$; Placebo pre: $\mathrm{M}=6.18, \mathrm{SD}=5.48$; Placebo post $M=41.32, S D=26.06$; Losartan pre: $M=8.90, S D=8.38$; Losartan post: $M=35.87, S D=18.97)[21,22]$. There was neither a main effect of group nor a group $\times$ time interaction (both $F(1,38)$ $<1.24$, both $p>0.272$, both $\eta_{\mathrm{p}}{ }^{2}<0.032$ ).

Peritraumatic Processing Task

Manipulation check. (a) Accuracy: Accuracy was the number of pictures correctly categorised as belonging to the trauma film or not (negative match). A $2 \times 2$ mixed-model ANOVA with group as between-factor (placebo, losartan) and picture type as withinfactor (trauma, negative match) showed that accuracy was similarly high in both groups (placebo: $M=91 \%, S D=5 \%$; 
Table 1. Comparison of sociodemographic measures and attentional, clinical, and personality participant characteristics in the placebo and the losartan groups

Placebo $(N=20 ; \quad$ Losartan $(N=20 ; \quad p$ 13 female) $\quad 13$ female)

$M(S D) \quad M(S D)$

Sociodemographic measures

Age

National Adult

$26.0(7.9)$

$25.7(5.9)$

Reading Test

$117.7(5.3)$

$119.0(4.1)$

Years of education

$18.3(3.0)$

$21.4(16.3)$

Attentional, clinical and personality measures

Attentional

$56.4(6.4)$

$58.9(7.8)$

Control Scale

Beck Depression

$2.4(3.3)$

$1.8(2.2)$

Behavioural

Inhibition Scale

Behavioural

$15.1(4.1)$

$15.1(4.1)$

$24.1(5.3)$

$25.0(5.1)$

Activation Scale

Anxiety Sensitivity Index

Spielberger's Trait

Anxiety Inventory

Trauma History Checklist

Post-traumatic

Cognition Inventory

EPQ - Psychosis

EPQ - Neuroticism

$E P Q$ - Extraversion

EPQ - Lie

$14.0(10.4)$

$16.5(14.0)$

$35.1(7.8)$

$33.1(5.2)$

$0.7(1.1)$

$1.1(1.7)$

$64.6(19.9)$

$70.8(21.3)$

$2.7(2.3)$

$3.0(2.7)$

$7.5(5.4)$

$15.5(3.1)$

$10.2(5.0)$

$5.8(4.1)$

$15.5(4.4)$

$9.2(3.8)$

$E P Q$ Eysenck Personality Inventory

losartan: $\mathrm{M}=92 \%, \mathrm{SD}=6 \%$; main effect group/group $\times$ condition interaction: both $F^{\prime} s<1.44$, both $p>0.238$, both $\left.\eta_{p}{ }^{2}>0.036\right)$. In both groups, participants were more accurate in correctly categorising negative match pictures as not belonging to the films compared to categorising trauma pictures as belonging to the films (main effect picture type: $F(1,38)=9.89, p=0.003, \eta_{\mathrm{p}}{ }^{2}=$ 0.206; Fig. 2a). (b) Reaction times (RT): A $2 \times 2 \times 2$ mixed-model ANOVA with the factors drug group, picture type (trauma, negative match), and accuracy (correct, incorrect) revealed no main effects $\left(p^{\prime} s>0.063, \eta_{p}{ }^{2}>0.122\right)$ nor any interaction effects between the factors (all $p^{\prime} s>0.102, \eta_{p}{ }^{2}>0.096$ ). Incorrect trials were excluded from subsequent analyses.

Data-driven processing. RTs in recognising the pictures' contents were analysed using a 2 drug group $\times 2$ picture type (trauma, negative match) mixed-model ANOVA. Contrary to our expectations, both groups were faster in recognising trauma-film pictures compared to negative-match pictures (main effect picture type: $F$ $(1,38)=267.56, p<0.001, \eta_{p}{ }^{2}=0.876$; main effect group/group $\times$ condition interaction: both $F^{\prime} s(1,38)<0.83$, both $p^{\prime} s>0.369$, both $\left.\eta_{\mathrm{p}}{ }^{2}<0.021\right)$, indicating that losartan had no influence on datadriven processing. A potential speed-accuracy trade-off seems unlikely, since accuracy in both conditions was very high and disproportionate to the differences in RTs (Fig. 2b).

Correlations RTs and reported details. For the trauma pictures, correlational analyses revealed no association between RTs and level of reported detail $(r(40)=0.088, p=0.591$; for individual stimuli all $p^{\prime} s>0.109$ ), indicating that participants indeed reported more detail because they retrieved additional memory information, rather than simply perceiving more information due to longer picture presentations. In contrast, for negative-match pictures, there was a significant correlation between RT and reported level of detail $(r(40)=0.419, p=0.007)$. Since this condition reflects purely perceptive processing, it is reasonable to assume that participants perceived more details when the picture was presented for a longer period of time. Thus, only in the negative-match condition we controlled for RTs in the subsequent analyses (for scatterplots, see Supplementary Fig. S1A).

Contextual processing. Level of reported detail was compared between groups using an independent-samples $t$-tests for the trauma pictures and an ANCOVA in the negative-match pictures (controlling for RTs), since they reflect different cognitive processes as indicated in our correlational analyses and therefore cannot be compared directly. Regarding the trauma pictures, the losartan group reported significantly more detail than the placebo group $(t(38)=-3.75, p=0.001, d=-1.15$; Fig. $2 c)$, indicating deeper contextual processing of trauma images after drug administration compared to placebo. In the negative-match pictures, the losartan group perceived more detail compared to the placebo group, when controlling for RTs $(F(1,37)=6.61, p=$ $0.014, \eta_{p}{ }^{2}=0.152$; Fig. 2d).

Peritraumatic HR and HRV during film

HR was not recorded for one losartan participant due to technical failure. Two further losartan participants were excluded from analyses due to extreme values (deviation > 3 SD above mean). While participants' HRs were comparable across groups at drugpeak level prior to film presentation $(t(38)=1.05, p=0.300, d=$ 0.33), losartan compared to placebo participants showed lower HR during film presentation $(t(26.14)=2.26, p=0.032, d=0.44)$, indicating lower arousal levels (Fig. 3a; Fig. 3b for minute-byminute analysis). The drug group also showed significantly lower LF/HF ratios than controls during film presentation $(t(29.95)=$ 2.74, $p=0.010, d=0.81$ ), suggesting higher HRV and higher parasympathetic compared to sympathetic activation during trauma after losartan administration (Fig. 3c).

Correlational analyses exploring the relationship between HR during film and contextual processing showed that higher HR was associated with fewer reported details in the trauma picture condition $(r(37)=-0.363, p=0.027)$. For negative-match pictures, we calculated the semi-partial correlation between HR during film and detail perception, controlling for the effect of RT on detail perception. However, there was no significant correlation, indicating that perceptual processing of peripheral trauma-cues seems unrelated by higher arousal levels $(r(37)=-0.308, p=$ 0.064 ; Figure S1B). We obtained a similar pattern when conducting the same correlation analyses between detail retrieval and LF/HF ratio (correlation trauma: $r(37)=-0.411, p=0.012$; semi-partial correlation negative match: $r(37)=0.274, p=0.100)$; Figure S1C). We repeated relevant analyses controlling for baseline HR and results were comparable (Supplementary Material).

\section{Trauma symptom assessment}

PTCl pre- and post-film was analysed using a 2 time (pre- and post-film) $\times 2$ drug group mixed-model ANOVA. There was neither a significant main effect of group $(F(1,38)=0.938, p=0.339$, $\left.\eta_{\mathrm{p}}{ }^{2}=0.024\right)$ or time $\left(F(1,38)=4.00, p=0.053, \eta_{\mathrm{p}}{ }^{2}<0.095\right)$ nor an interaction effect $\left(F(1,38)=0.011, p=0.981, \eta_{\mathrm{p}}^{2}<0.000\right)$, indicating no drug effects on post-traumatic cognitions. At follow-up, independent-samples $t$-tests revealed that losartan, compared to placebo, had neither an effect on PTCl scores nor on general PTSD symptoms indicated by the PCL-5 sum and cluster scores (all $p$ 's > 0.17).

All participants completed and returned the intrusion diary. Independent-samples $t$-tests revealed no group difference between losartan and placebo regarding intrusion frequency 
A Accuracy

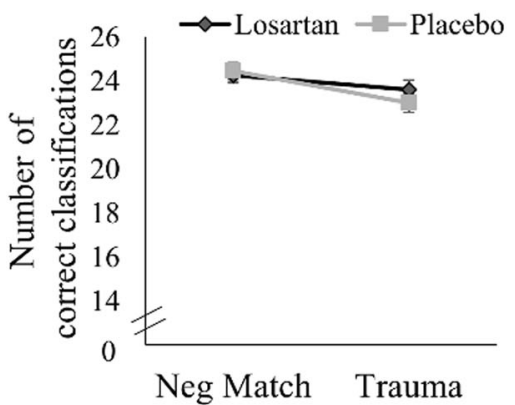

C Contextual-Processing: Trauma

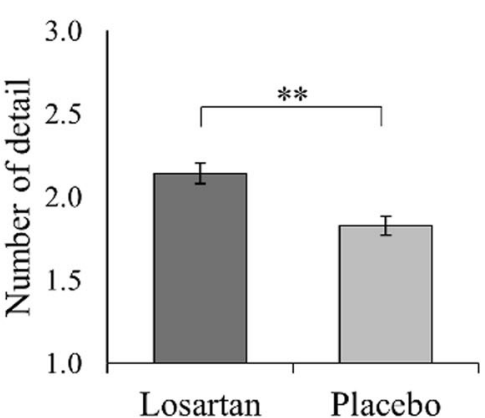

\section{B Data-driven processing}

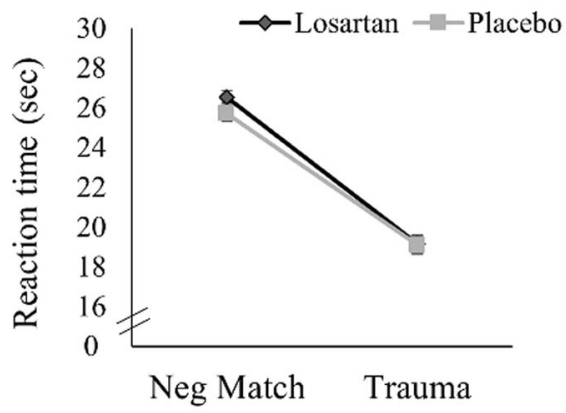

D Control: Neg-Match

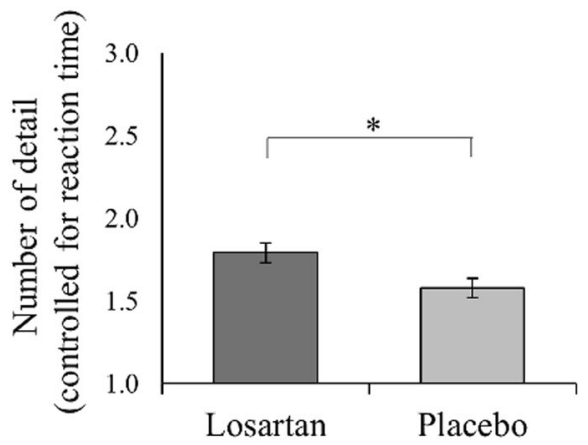

Fig. 2 Performance on the peritraumatic processing task as an indicator for data driven and contextual processing (mean, standard errors). a Accuracy. Total number of pictures correctly categorised as belonging to the trauma film versus new pictures (i.e. negative match). Across both groups, participants were better in correctly categorising the negative-matched pictures as not belonging to the trauma films, compared to categorising the trauma-film pictures as part of the films. b Data-driven processing as reaction time to recognise picture content, with faster response reflecting picture recognition at blurrier picture levels during the task. Both groups were faster in recognising the general contents of the trauma pictures compared to the general contents of the negative-match picture. c Contextual processing reflected by the mean reported details per picture for trauma pictures. Participants could include information which was not depicted in the presented picture itself but which they deliberately retrieved based on their trauma-film memory. The losartan group scored higher than the placebo group, indicating an advantage in contextual processing. d Negative match: Perceived level of detail in the negative-match pictures, controlled for reaction time. This reflects pure perceptual processes, since all pictures were new to the participants and no memory effects could interfere. The losartan compared to placebo group scored higher, indicating visual processing advantages for peripheral trauma-cues. Note that the $y$ axis depicts standardised residuals. ${ }^{*} p<0.05,{ }^{* *} p<0.01$

(placebo: $\mathrm{M}=4.25, \mathrm{SD}=3.99$; losartan: $\mathrm{M}=5.20, \mathrm{SD}=4.11, t(38)=$ $-0.74, p=0.46, d=-0.23$ ) and intrusion distress (placebo: $\mathrm{M}=$ 40.75, $S D=19.53$; losartan: $M=34.56, S D=20.55, t(38)=0.98$, $p=0.34, d=0.31$.

\section{Explicit memory}

Losartan administration did not affect explicit memory for traumafilm contents assessed at follow-up, since the two groups did not differ on their overall memory score (placebo: $M=18.15, S D=$ 2.77; losartan: $\mathrm{M}=18.88, \mathrm{SD}=2.60 ; t(38)=-0.86, p=0.396, d=$ $-0.27)$.

\section{DISCUSSION}

The present study aimed to investigate the effect of the $A T_{1}$ receptor antagonist losartan on data-driven and contextual information processing during analogue trauma exposure, mechanisms previously indicated in the development of posttraumatic stress symptoms [10-13]. In line with our prediction, losartan, unlike placebo, led to an increase in contextual processing, reflected in greater retrieval of contextual details for trauma pictures. However, losartan had no influence on datadriven processing, and-contrary to our expectation-the drugrelated increase in contextual processing was not accompanied by a decrease in analogue PTSD symptom severity such as intrusion frequency. While there were no group differences in HR immediately before trauma-film presentation, losartan compared to placebo prevented an increase in HR in response to the films, and the losartan group showed greater HRV during film exposure, reflecting lower sympathetic activity. This suggests that the drug may have reduced autonomic stress reactivity in response to the threatening material, and thus preserved information processing during trauma exposure. In line with this, we found a negative correlation between both HR and HRV and level of reported details for the trauma images only. The drug also enhanced the perceptual processing of peripheral trauma-cues, reflected in perceiving a greater level of detail in blurred negative-match images compared to placebo.

These findings provide first evidence that single-dose losartan prevents an HR increase and an elevated sympathetic activity during an analogue trauma event, which in turn might facilitate contextual processing, bringing forward a potential mechanism by which the drug might prevent the disorder's onset. These results extend previous work in humans where renin-angiotensin drugs were associated with reduced PTSD symptom development, and shed light on the specific cognitive mechanisms underlying such findings [6].

The formation of contextual memory representations during a traumatic event is hippocampus-dependent $[10,13]$. During stress, however, hippocampal memory formation is impaired in favour of low-level stimulus-response learning [53], resulting in fragmented contextual memory formation and thus PTSD symptom 

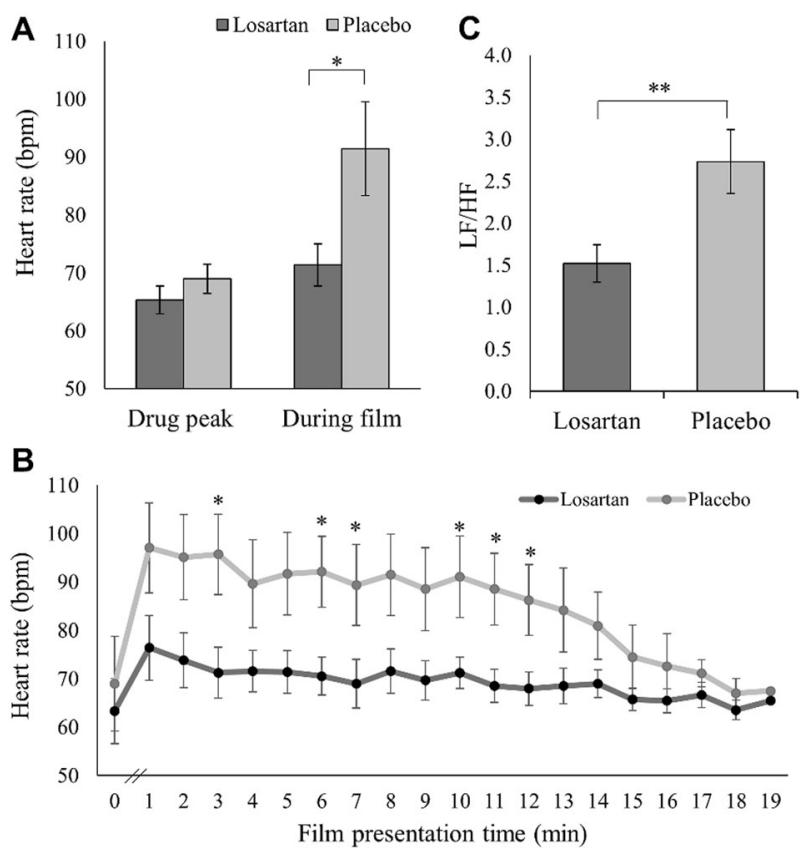

Fig. 3 Trauma-film-related changes in heart rate (HR) across both groups (mean, standard errors). a HR before and during trauma-film presentation. During film presentation, HR is significantly increased in the placebo but not in the losartan group. b Minute-by-minute analysis of mean HR. While there was no group difference at baseline just before film presentation (drug-peak level), HR immediately increased in response to the clips in the placebo group but returned to baseline level towards the end of the film. In the losartan group, HR remained at baseline level throughout film presentation. Groups differ at minutes $3,6,7,10,11,12(p<0.05)$, respectively. Additionally, there is a non-significant trend in minute 1 $(p=0.091)$, minute $2(p=0.072)$, minute $4(p=0.075)$, minute $5(p=$ $0.054)$, minute $8(p=0.050)$, minute $9(p=0.058)$ and minute 13 $(p=0.063)$. From minute 14 onwards, all $p$ 's $>0.251$. c Heart Rate Variability (HRV). Low frequency/High frequency ratio (LF/HF) was calculated using time-frequency-domain analyses in the Fast Fourier Transform spectrum. LF/HF is significantly lower in the losartan compared to the placebo group, reflecting higher HRV manifesting in more parasympathetic activity in relation to sympathetic activity. ${ }^{*} p<0.05 ;{ }^{* *} p=0.01$

development [10]. On the one hand, losartan might have acted indirectly on these mechanisms in that it may have prevented an HR increase and elevated sympathetic response during the films. As a result, participants might have experienced lower levels of stress that could have interfered with hippocampal processing. Alternatively, since angiotensin-II influences hippocampusdependent learning and memory processes [24-26], losartan may have acted directly by enhancing hippocampal activity, thus enhancing contextual memory formation, which then could have resulted in reduced stress reactions indicated by a decreased HR and increased HRV. The data and paradigm established here provide an ideal platform to further investigate the effect of losartan on neurobiological markers of stress, including cortisol or norepinephrine responses to traumatic stress, as well as hippocampal plasticity, to further disentangle the drug's mechanisms of action underlying the neurocognitive effects observed in this study.

For the negative-match pictures, general threat was recognised equally across both groups, indicated by similar RTs. However, losartan led to perceiving more detail at blurrier picture levels, meaning that losartan administration enhanced visual discrimination of peripheral trauma-cues in a complex scene. PTSD patients are known to show difficulty in distinguishing safe from traumatic stimuli aspects in response to trauma-related cues, which can lead to re-experiencing symptoms [12, 13]. This is supported by conditioning studies where fear acquisition and extinction took place in different contexts ('danger' context in acquisition and 'safe' context in extinction), showing that PTSD patients, compared to trauma-exposed controls without PTSD, exhibited greater fear responses in the safe context the day after extinction $[54,55]$. Similarly, previous studies found that single-dose losartan administration led to improved extinction in the safe context in an animal model [7], and improved threat-safety differentiation in response to facial stimuli in the amygdala in high-trait anxious humans [56]. Possibly, losartan prevents the development of posttraumatic symptoms by enhancing the processing of traumarelated cues and distinguishing them successfully from the traumatic event. This mechanism would have important implications for the combination of this drug with exposure therapy, where it might boost clinical effects by enhancing the integration of context information and the processing of safety information.

Considering recent studies into the potential of the blood pressure drug propranolol in improving anxiety treatments, one might wonder how research into losartan adds to this debate. Propranolol is a $\beta$-adrenergic antagonist thought to reduce anxiety by inhibiting reconsolidation after brief fear memory activation [57]. However, this procedure remains time sensitive and difficult to control in clinical practice, and efficacy trials in anxiety patients have led to mixed effects [57]. Propranolol has also been shown to have no lasting or even detrimental effects on fear extinction and exposure therapy in humans [58-61], possibly by interfering with threat processing crucial to exposure success: while increased amygdala sensitivity before exposure predicts improved clinical outcome, single-dose propranolol dampens amygdala response [62]. In contrast, losartan appears to have sustained effects on fear extinction [7] and to maintain amygdala threat response [56], suggesting mechanisms of action that might -different from propranolol-synergistically overlap with exposure.

While these findings shed further light on how losartan might affect emotional processing, some limitations regarding study design and interpretability of results require discussion. First, we found no association between the effects of losartan on peritraumatic processing and HR versus analogue PTSD symptoms, limiting our ability to conclude cognitive mechanisms of PTSD prevention. It is possible that while single administration of losartan leads to relevant cognitive and physiological changes, its effects are too small to affect symptom levels. Importantly, in the cohort study reporting preventive effects on PTSD symptom development, patients were prescribed regular drug intake at higher doses, suggesting that symptom effects might only be seen with more regular administration [6]. Second, we did not find an effect of losartan on explicit memory recognition, even though it affected contextual processing. This might be due to ceiling effects in a simple multiple-choice format (70\% accuracy across groups), or very different retrieval demands (being able to guess or recognise versus having to freely recall). Further, since we only included trauma film or trauma-related negative-match stimuli, we cannot rule out that losartan merely leads to cognitive enhancement in general, as opposed to traumaspecific effects. Future designs should thus include traumaunrelated negative and neutral pictures to further disentangle these potential effects.

In sum, the present study provides first evidence that losartan influences the way traumatic events are processed. Our results highlight stable peritraumatic physiological arousal, an increase in contextual processing, and an enhancement in visual discrimination of peripheral trauma-cues as promising mechanistic candidates of PTSD symptom prevention, and they point towards important further directions for systematic follow-up 
research, especially regarding the therapeutic application of losartan.

\section{FUNDING AND DISCLOSURE}

This research was funded by a MQ: Transforming Mental Health fellowship awarded to A.R. (MQ14F192) and a post-doctoral scholarship of the Daimler and Benz Foundation awarded to M.L.W. (32-12/4). L.S. was supported by an Erasmus scholarship. The authors declare no competing interest.

\section{ADDITIONAL INFORMATION}

Supplementary Information accompanies this paper at (https://doi.org/10.1038/ s41386-019-0553-y)

Publisher's note Springer Nature remains neutral with regard to jurisdictional claims in published maps and institutional affiliations.

\section{REFERENCES}

1. Hidalgo RB, Davidson JR. Posttraumatic stress disorder: epidemiology and healthrelated considerations. J Clin Psychiatry. 2000;61:5-13.

2. Kessler RC. Posttraumatic stress disorder: the burden to the individual and to society. J Clin Psychiatry. 2000;61:4-12. discussion 13-4.

3. Pulcu E, Shkreli L, Holst CG, Woud ML, Craske MG, Browning M, et al. The effects of the angiotensin II receptor antagonist losartan on appetitive versus aversive learning: a randomized controlled trial. Biol Psychiatry. 2019. https://doi.org/ 10.1016/j.biopsych.2019.04.010.

4. Reinecke A, Harmer C. A cognitive-neuropsychological account of treatment action in anxiety: can we augment clinical efficacy? Psychopathol Rev. 2016;3:pr035113 https://doi.org/10.5127/pr.035113.

5. Marinzalda MdIA, Pérez PA, Gargiulo PA, Casarsa BS, Bregonzio C, Baiardi G. Fearpotentiated behaviour is modulated by central amygdala angiotensin II AT1 receptors stimulation. Biomed Res Int. 2014;2014:183248. https://doi.org/10.1155/ 2014/183248.

6. Khoury NM, Marvar PJ, Gillespie CF, Wingo A, Schwartz A, Bradley B. et al. The renin-angiotensin pathway in posttraumatic stress disorder: angiotensinconverting enzyme inhibitors and angiotensin receptor blockers are associated with fewer traumatic stress symptoms. J Clin Psychiatry. 2012;73:849-55. https:// doi.org/10.4088/JCP.11m07316.

7. Marvar PJ, Goodman J, Fuchs S, Choi DC, Banerjee S, Ressler KJ. Angiotensin type 1 receptor inhibition enhances the extinction of fear memory. Biol Psychiatry. 2014;75:864-72. https://doi.org/10.1016/j.biopsych.2013.08.024.

8. von Bohlen und Halbach O, Albrecht D. The CNS renin-angiotensin system. Cell Tissue Res. 2006;326:599-616. https://doi.org/10.1007/s00441-006-0190-8.

9. Wright JW, Harding JW. The brain renin-angiotensin system: a diversity of functions and implications for CNS diseases. Pflug Arch. 2013;465:133-51. https://doi. org/10.1007/s00424-012-1102-2.

10. Brewin CR, Gregory JD, Lipton M, Burgess N. Intrusive images in psychological disorders: characteristics, neural mechanisms, and treatment implications. Psychol Rev. 2010;117:210-32. https://doi.org/10.1037/a0018113.

11. Brewin $C R$, Dalgleish $T$, Joseph $S$. A dual representation theory of posttraumatic stress disorder. Psychol Rev. 1996;103:670-86. https://doi.org/10.1037/0033295X.103.4.670.

12. Ehlers A, Clark DM. A cognitive model of posttraumatic stress disorder. Behav Res Ther. 2000;38:319-45. https://doi.org/10.1016/S0005-7967(99)00123-0.

13. Brewin CR. A cognitive neuroscience account of posttraumatic stress disorder and its treatment. Behav Res Ther. 2001;39:373-93. https://doi.org/10.1016/ S0005-7967(00)00087-5.

14. Ehlers A, Michael T, Chen YP, Payne E, Shan S. Enhanced perceptual priming for neutral stimuli in a traumatic context: a pathway to intrusive memories?. Memory. 2006;14:316-28. https://doi.org/10.1080/09658210500305876.

15. Michael T, Ehlers A. Enhanced perceptual priming for neutral stimuli occurring in a traumatic context: two experimental investigations. Behav Res Ther. 2007;45:341-58. https://doi.org/10.1016/j.brat.2006.03.012.

16. Kleim B, Ehring T, Ehlers A. Perceptual processing advantages for trauma-related visual cues in post-traumatic stress disorder. Psychol Med. 2012;42:173-81. https://doi.org/10.1017/S0033291711001048.

17. Kindt $M$, van den Hout $M$, Arntz A, Drost J. The influence of data-driven versus conceptually-driven processing on the development of PTSD-like symptoms. J Behav Ther Exp Psychiatry. 2008;39:546-57. https://doi.org/10.1016/j. jbtep.2007.12.003.
18. Halligan SL, Clark DM, Ehlers A. Cognitive processing, memory, and the development of PTSD symptoms: two experimental analogue studies. J Behav The Exp Psychiatry. 2002;33:73-89. https://doi.org/10.1016/S0005-7916(02)00014-9.

19. Pitman RK, Rasmusson AM, Koenen KC, Shin LM, Orr SP, Gilbertson MW. et al. Biological studies of post-traumatic stress disorder. Nat Rev Neurosci. 2012;13:769-87. https://doi.org/10.1038/nrn3339.

20. Holmes EA, Brewin CR, Hennessy RG. Trauma films, information processing, and intrusive memory development. J Exp Psychol Gen. 2004;133:3-22. https://doi. org/10.1037/0096-3445.133.1.3.

21. Woud ML, Holmes EA, Postma P, Dalgleish T, Mackintosh B. Ameliorating intrusive memories of distressing experiences using computerized reappraisal training. Emotion. 2012;12:778-84. https://doi.org/10.1037/a0024992.

22. Woud ML, Postma $P$, Holmes EA, Mackintosh B. Reducing analogue trauma symptoms by computerized reappraisal training-considering a cognitive prophylaxis? J Behav Ther Exp Psychiatry. 2013;44:312-5. https://doi.org/10.1016/j. jbtep.2013.01.003.

23. Llano López LH, Caif F, García S, Fraile M, Landa Al, Baiardi G. et al. Anxiolytic-like effect of losartan injected into amygdala of the acutely stressed rats. Pharmacol Rep. 2012;64:54-63. https://doi.org/10.1016/S1734-1140(12)70730-2.

24. Bild W, Hritcu L, Stefanescu C, Ciobica A. Inhibition of central angiotensin II enhances memory function and reduces oxidative stress status in rat hippocampus. Prog Neuropsychopharmacol Biol Psychiatry. 2013;43:79-88. https://doi. org/10.1016/j.pnpbp.2012.12.009.

25. Tashev R, Stefanova M. Hippocampal asymmetry in angiotensin II modulatory effects on learning and memory in rats. Acta Neurobiol Exp (Wars). 2015;75:48-59.

26. Kim M-S, Lee G-H, Kim Y-M, Lee B-W, Nam HY, Sim U-C, et al. Angiotensin Il causes apoptosis of adult hippocampal neural stem cells and memory impairment through the action on AMPK-PGC1a signaling in heart failure. Stem Cells Transl Med. 2017:6:1491-503. https://doi.org/10.1002/sctm.16-0382

27. Bedi US, Arora R. Cardiovascular manifestations of posttraumatic stress disorder. J Natl Med Assoc. 2007;99:642-9.

28. Gould NF, McKibben JB, Hall R, Corry NH, Amoyal NA, Mason ST. et al. Peritraumatic heart rate and posttraumatic stress disorder in patients with severe burns. $J$ Clin Psychiatry. 2011;72:539-47. https://doi.org/10.4088/JCP.09m05405blu.

29. Elsesser K, Sartory G, Tackenberg A. Initial symptoms and reactions to traumarelated stimuli and the development of posttraumatic stress disorder. Depress Anxiety. 2005;21:61-70. https://doi.org/10.1002/da.20047.

30. Shah AJ, Lampert R, Goldberg J, Veledar E, Bremner JD, Vaccarino V. Posttraumatic stress disorder and impaired autonomic modulation in male twins. Biol Psychiatry. 2013;73:1103-10. https://doi.org/10.1016/j.biopsych.2013.01.019.

31. Davern PJ, Chen D, Head GA, Chavez CA, Walther T, Mayorov DN. Role of angiotensin II Type $1 \mathrm{~A}$ receptors in cardiovascular reactivity and neuronal activation after aversive stress in mice. Hypertension. 2009;54:1262-8. https://doi. org/10.1161/HYPERTENSIONAHA.109.139741.

32. Matteo R, de, Head GA, Mayorov DN. Angiotensin II in dorsomedial hypothalamus modulates cardiovascular arousal caused by stress but not feeding in rabbits. Am J Physiol Regul Integr Comp Physiol. 2006;290:R257-64. https://doi.org/10.1152/ ajpregu.00372.2005.

33. First MB, Williams JBW, Karg RS, Spitzer RL. Structured clinical interview for DSM-5 disorders: Scid-5-cv: clinician version. Arlington, VA: American Psychiatric Association Publishing; 2016.

34. Nelson HE. The National Adult Reading Test (NART): test manual. Windsor: NFERNelson; 1982

35. Eysenck HJ, Eysenck SBG. Manual of the Eysenck personality questionnaire (junior and adult). Kent, UK: Hodder and Stoughton; 1975.

36. Foa EB, Ehlers A, Clark DM, Tolin DF, Orsillo SM. The Posttraumatic Cognitions Inventory (PTCl): development and validation. Psychological Assess. 1999;11:303-14. https://doi.org/10.1037/1040-3590.11.3.303.

37. Taylor S, Cox BJ. An Expanded Anxiety Sensitivity Index. J Anxiety Disord 1998;12:463-83. https://doi.org/10.1016/S0887-6185(98)00028-0.

38. Beck AT, Steer RA, Brown GK. Beck Depression Inventory-II manual. 2nd ed. San Antonio, TX: The Psychological Corporation; 1996.

39. Spielberger CD, Gorusch RL, Lushene R, Vagg PR, Jacobs GA. Manual for the StateTrait Anxiety Inventory. Palo Alto, CA: Consulting Psychologists Press; 1970.

40. Derryberry D, Reed MA. Anxiety-related attentional biases and their regulation by attentional control. J Abnorm Psychol. 2002;111:225-36. https://doi.org/10.1037// 0021-843X.111.2.225.

41. Carver CS, White TL. Behavioral inhibition, behavioral activation, and affective responses to impending reward and punishment: The BIS/BAS Scales. J Personal Soc Psychol. 1994;67:319-33. https://doi.org/10.1037/0022-3514.67.2.319.

42. Lo M-W, Goldberg MR, McCrea JB, Lu H, Furtek Cl, Bjornsson TD. Pharmacokinetics of losartan, an angiotensin II receptor antagonist, and its active metabolite EXP3174 in humans. Clin Pharm Ther. 1995;58:641-9. https://doi.org/10.1016/ 0009-9236(95)90020-9. 
43. Ohtawa M, Takayama F, Saitoh K, Yoshinaga T, Nakashima M. Pharmacokinetics and biochemical efficacy after single and multiple oral administration of losartan, an orally active nonpeptide angiotensin II receptor antagonist, in humans. Br J Clin Pharmacol. 1993;35:290-7. https://doi.org/10.1111/j.1365-2125.1993.tb05696.x.

44. Weathers FW, Litz BT, Keane TM, Palmieri PA, Marx BP, Schnurr PP. PTSD Checklist for DSM-5 (PCL-5). Boston, MA: National Center for PTSD; 2013.

45. James EL, Lau-Zhu A, Clark IA, Visser RM, Hagenaars MA, Holmes EA. The trauma film paradigm as an experimental psychopathology model of psychological trauma: Intrusive memories and beyond. Clin Psychol Rev. 2016;47:106-42. https://doi.org/10.1016/j.cpr.2016.04.010.

46. American Psychiatric Association. Diagnostic and statistical manual of mental disorders. 5th ed. Washington, DC: American Psychiatric Association; 2013.

47. James EL, Bonsall MB, Hoppitt L, Tunbridge EM, Geddes JR, Milton AL. et al. Computer game play reduces intrusive memories of experimental trauma via reconsolidation-update mechanisms. Psychol Sci. 2015;26:1201-15. https://doi. org/10.1177/0956797615583071.

48. Gilder M, Ramsbottom R. Measures of cardiac autonomic control in women with differing volumes of physical activity. J Sports Sci. 2008;26:781-6. https://doi.org/ 10.1080/02640410701836895.

49. Lang PJ, Bradley MM, Cuthbert BN. The international affective picture system. FL: NIMH Centre for the Study of Emotion and Attention, University of Florida; 1997.

50. Krans J, Pearson DG, Maier B, Moulds ML. Contextual representations of negative images modulate intrusion frequency in an intrusion provocation paradigm. J Behav Ther Exp Psychiatry. 2016;53:52-8. https://doi.org/10.1016/j. jbtep.2015.09.004.

51. Woud ML, Cwik JC, Blackwell SE, Kleim B, Holmes EA, Adolph D. et al. Does napping enhance the effects of cognitive bias modification-appraisal training? An experimental study. PLoS ONE. 2018;13:e0192837 https://doi.org/10.1371/journal. pone.0192837.

52. Woud ML, Heeren A, Shkreli L, Meyer T, Egeri L, Cwik JC. et al. Investigating the effect of proactive interference control training on intrusive memories. Eur J Psychotraumatol. 2019;10:1611092 https://doi.org/10.1080/20008198.2019.1611092.
53. Schwabe L, Wolf OT. Stress modulates the engagement of multiple memory systems in classification learning. J Neurosci. 2013;32:11042-9. https://doi.org/ 10.1523/JNEUROSCI.1484-12.2012.

54. Garfinkel SN, Abelson JL, King AP, Sripada RK, Wang X, Gaines LM. et al. Impaired contextual modulation of memories in PTSD: an fMRI and psychophysiological study of extinction retention and fear renewal. J Neurosci. 2014;34:13435-43. https://doi.org/10.1523/JNEUROSCl.4287-13.2014.

55. Milad MR, Pitman RK, Ellis CB, Gold AL, Shin LM, Lasko NB. et al. Neurobiological basis of failure to recall extinction memory in posttraumatic stress disorder. Biol Psychiatry. 2009;66:1075-82. https://doi.org/10.1016/j.biopsych.2009.06.026.

56. Reinecke A, Browning M, Klein Breteler J, Kappelmann N, Ressler KJ, Harmer CJ, et al. Angiotensin regulation of amygdala response to threat in high-trait-anxiety individuals. Biol Psychiatry Cogn Neurosci Neuroimaging. 2018. https://doi.org/ 10.1016/j.bpsc.2018.05.007.

57. Kindt $M$. The surprising subtleties of changing fear memory: a challenge for translational science. Philos Trans R Soc Lond B Biol Sci. 2018. https://doi.org/ 10.1098/rstb.2017.0033.

58. Brunet A, Orr SP, Tremblay J, Robertson K, Nader K, Pitman RK. Effect of postretrieval propranolol on psychophysiologic responding during subsequent scriptdriven traumatic imagery in post-traumatic stress disorder. J Psychiatr Res. 2008;42:503-6. https://doi.org/10.1016/j.jpsychires.2007.05.006.

59. Chalkia A, Weermeijer J, van Oudenhove L, Beckers T. Acute but not permanent effects of propranolol on fear memory expression in humans. Front Hum Neurosci. 2019;13:51. https://doi.org/10.3389/fnhum.2019.00051.

60. Hafner J, Milton F. The influence of propranolol on the exposure in vivo of agoraphobics. Psychol Med. 1977;7:419-25. https://doi.org/10.1017/ S0033291700004384.

61. Meyerbroeker K, Morina N, Emmelkamp PMG. Enhancement of exposure therapy in participants with specific phobia: a randomized controlled trial comparing yohimbine, propranolol and placebo. J Anxiety Disord. 2018;57:48-56. https://doi. org/10.1016/j.janxdis.2018.05.001.

62. Hurlemann R, Walter H, Rehme AK, Kukolja J, Santoro SC, Schmidt C. et al. Human amygdala reactivity is diminished by the $\beta$-noradrenergic antagonist propranolol. Psychol Med. 2010;40:1839-48. https://doi.org/10.1017/S0033291709992376. 